\title{
MALE CIRCUMCISION SHOULD BE PROMOTED IN DEVELOPING COUNTRIES AS A MAJOR MEANS OF HIV PREVENTION
}

\author{
Ali F, Vishal M \\ Institute of public Health, University of Heidelberg, Im Neuenheimer Feld 365, Heidelberg, Germany
}

\section{INTRODUCTION}

Male circumcision is a religious practice in some of the countries in Asia and Africa. In the Muslim community it is particularly a very important ceremony where the fore skin of the penis is cut to remove normally before the fifth birthday of the boys particularly in Indian Muslim community. There are some other ethnic groups that perform this practice across Africa. However in the countries such as USA, Canada, Australia and South Korea it is performed irrespective of the culture, religion and ethnicity. ${ }^{1}$

Male circumcision is deeply rooted in the culture and moral values of many communities in the developing world and hence remains a sensitive issue when it comes to link it with the issues of reproductive health and sexuality. The issue becomes more sensitive in the wake of STIs and HIVIAIDS. Linking the transmission of HIV and uncircumcised male has witnessed intense resistance and doubt and silence over the years. This is reflected well in less availability of public health interventions and information on this issue. ${ }^{2}$

Almost 20 years have passed when Cameron and colleagues presented a study that showed that there is 8 times more risk for contracting HIV -1 among uncircumcised males. ${ }^{3}$ In this article I discuss the

\footnotetext{
Correspondence to

Mr. Farhad Ali,

Institute of public Health,

University of Heidelberg,

Im Neuenheimer Feld 365, Heidelberg,

Germany

E-mail: farhadali1@gmail.com
}

promotion of male circumcision in developing countries as a major means of HIV prevention.

\section{DISCUSSION}

\begin{tabular}{|c|c|c|c|}
\hline S. No. & Country & $\begin{array}{l}\text { Per cent of } \\
\text { circumcised } \\
\text { male }\end{array}$ & $\begin{array}{l}\text { HIV } \\
\text { Seroprevalence } \\
\text { rate in low risk } \\
\text { groups }\end{array}$ \\
\hline 1. & Zimbabwe & $10.1 \%$ & $19.7 \%$ \\
\hline 2. & Bostwana & $11.0 \%$ & $41.6 \%$ \\
\hline 3. & Namibia & $21 \%$ & $16 \%$ \\
\hline 4. & Thailand & $13 \%$ & $1.1 \%$ \\
\hline 5. & Phillies & $93 \%$ & $0.3 \%$ \\
\hline 6. & Kenya & $83.0 \%$ & $10.5 \%$ \\
\hline 7. & Congo & $90 \%$ & $3.1 \%$ \\
\hline 8. & Cameroon & $>80 \%$ & $6.9 \%$ \\
\hline
\end{tabular}

Let us have a look of the figures presented in table No.1. If we compare the figures by segregating the data into two categories of those countries where the per cent male circumcised is more than 80 per cent and less than 20 per cent and compare it with the prevalence rate of HIV seropositivity, we find a beneficial effect of male circumcision in terms of low HIV seropositivity.

Though there are different conditions prevailing in these countries with respect to health system, risk factors, socio-economic and cultural values and norms and interventions on HIVIAIDS even though the seropositivity differences are worth noticing. Daniel et al also argues on the similar lines in their study on male circumcision and HIV infection. ${ }^{1}$ 
There are several studies that explain uncircumcised males are more prone to contract HIV and other pathogen through the fore skin. Simonsen et al reported as early as in the year 1988 that fore skin contain higher density of primary target cells that facilitate sexual transmission of HIV than cervical, vaginal and rectal mucosa. ${ }^{4}$ Patterson et al in the year 2002 reported that under in vitro culture, human foreskin is highly susceptible to HIV $-1 .{ }^{5}$ Hussian et al reported that in histopathological studies, epithelium of human foreskin provide main portal of entry for HIV-1 into the penis. ${ }^{6}$ The biological phenomenon reflects that there is a strong relationship between transmission of HIV -1 and presence of foreskin in the heterosexual activities suggesting that the removal of the foreskin of penis could be used as a public health strategy to prevent the transmission of HIV-1.

A study was conducted in India with 2298 HIV uninfected males attending clinics of sexually transmitted diseases. In this study it was found that the male circumcision was strongly protective against HIV $-1 .^{7}$ A similar trail was also conducted in Kenya with the young men in Kisumu for HIV prevention. In this trial also it was found that male circumcision significantly reduces the risk of acquisition of HIV. This study suggests that male circumcision services should be integrated with HIV prevention strategies. ${ }^{8}$

A trail was conducted in peri-urban settings in South Africa with 3274 men aged between 18-24 years. This trail recruited the participants from the general population and so loss to follow up was less in this trail. This also ensures the generalisability of the results of this trail. The results of the trial suggest that there is 60 per cent less relative risk of HIV in circumcised men. ${ }^{12}$

Seeing the benefits that male circumcision provides WHO and UNAIDS recommended that those men who want to get circumcised should be provided the procedure. These services should also be provided to those who do not wish to undergo HIV testing and irrespective of the HIV status of men. ${ }^{9}$

The benefits of male circumcision for men with respect to reduced risk of HIV transmission from the sex partners are evident but, the protection for the women is not obvious. Maria Wawer and colleagues conducted a clinical trial in Uganda. This trail was stopped in between as no benefit was found in terms of risk reduction for women whose HIV infected partners were circumcised. The data of this study suggest that the risk for the women increased whose partners resumed the sexual activities before the wound healing. ${ }^{10}$ No HIV intervention based on single stand alone approach would be effective unless a comprehensive package of various HIV prevention strategies are combined together. Male circumcision provides one such window of opportunity in long term benefits for both male and females considering the fact that if less number of male are infected the transmission in heterosexual relationships from male to female would be less. The benefits of couple counseling, constant and consistent use of condoms and couple testing for HIV can not be undermined. The benefits of circumcision in reducing men's risk of HIV are clear, and circumcision roll-out presents a unique opportunity to enhance HIV prevention services for both women and men. ${ }^{11}$

Male circumcision does not provide 100 per cent protection against the transmission of HIV and the use of condoms remains an important aspect of HIV prevention efforts. Similarly there are issues involved with the consistent and correct use of condoms. In the population the condom use is found to be inconsistent. So combining the benefits of condom use along with male circumcision provides a benefit that is worth noticing.

\section{MALE CIRCUMCISION AS A PUBLIC HEALTH INTERVENTION ACCEPTABILITY}

Circumcision is normally done for three main reasons. These are religious, ethnic and medical condition. ${ }^{13}$ The communities in Kenya, South Africa and Botswana have reported the acceptance of circumcision in the range of $51 \%$ to $61 \%$ among uncircumcised men. The biggest challenge in the intervention related to circumcision is that those communities that are at higher rick of HIV would be least accepting it as they are traditionally noncircumcising communities. There is also a stigma associated with circumcision in some communities and a systematic study needs to be carried out to understand the perception of these 
communities. The fear of pain, bleeding, reduced sexual pleasure and access to health services are some of the concern of the people. Related to the acceptability is the false perception of the people that circumcision would protect them from contracting HIV. This poses a huge problem and may cause a sense of false security. A study conducted in South Africa revealed that 30 per cent of circumcised men believe that they can safely have sex with multiple partners. ${ }^{14}$

\section{SAFETY}

Circumcision is a surgical process however in traditional settings it is being done by non-licensed practitioners. It can lead to complications if performed by untrained persons in nonmedical settings. A study done in Turkey reported that when circumcision was done in non-medical settings, 85 per cent of male adults faced complications. On the other hand when it was performed by licensed professionals only 2 per cent faced some kind of complications. ${ }^{15}$ From the above study it can be concluded that circumcision if performed in a medical settings by a trained professional can be a safer intervention.

\section{SOME OTHER CONSIDERATIONS}

There are some other issues that needs to be taken into consideration and these are legal issues, cultural sensitivity, human rights issues, ethical consideration when designing the interventions of male circumcision as a mean of HIV prevention. ${ }^{16}$

\section{CONCLUSION}

There are convincing evidences across various regions that male circumcision has the potential to reduce the transmission of HIV and thus reduce the reproductive rate of HIV in a population. Male circumcision could eventually be used as one of the strategies in controlling the spread of HIV within the comprehensive HIVIAIDS control programme in a developing country. The issues such as false security, safety period, infection control, cultural issues, women's safety issues, couple counseling for circumcision etc. should be carefully considered.

This strategy needs to be made affordable and safely available to the populations having high burden of the disease. The role of government, nongovernment organizations and funding agency become critically important in promoting this strategy.

\section{REFERENCES}

1. Daneil $T$ Halperin and Robert C Bailey, Male circumcision and HIV infection: 10 years and counting, Lancet 354 (1999), pp. 1813-1815.

2. PM Fleiss, FM Hodges and RS Van Howe, Immunological functions of the human prepuce, Sex Transm Infect 74 (1998), pp. 364-367.

3. DW Cameron, JN Simonsen and LJ D'Costa et.al., Female to male transmission of human immunodeficiency virus type -1: risk factors for seroconversion in men, Lancet 2 (1989), pp. 403-407.

4. JN Simonsen, DW Cameron and MN Gakinya et.al., Human immunodeficiency virus infection among men with sexually transmitted diseases, N Engl J Med 319 (1988), pp. 274-278.

5. BK Patterson, A. Landay and JN Siegel et.al., Susceptibility to human immunodeficiency virus-1 infection of human foreskin and cervical tissue grown in explant culture, Am J Pathol 161 (2002), pp. 867.

6. LA Hussain and T Lehner, Comparative investigation of Langerhans' cells and potential receptors for HIV in oral, genitourinary and rectal epithelia, Immunology 85 (1995), pp. 475.

7. SJ Reynolds, ME Shepherd, AR Risbud, RR Gangakhedkar, RS Brookmeyer, AD Divekar, SM Mehendale, RC Bollinger, Male circumcision and risk of HIV-1 and other sexually transmitted infections in India Lancet, 363 (2004), pp. 1039-1040.

8. RC Bailey, S Moses, CB Parker, K Agot, I Maclean, JN Krieger, CF Williams et.al., Ndinya-Achola JO, Male circumcision for HIV prevention in young men in Kisumu, Kenya: a randomised controlled trial Lancet, 369 (2007), pp. 643-656.

9. WHO/UNAIDS, New data on male circumcision and HIV prevention: policy and programme implications.

http://whqlibdoc. who.intpublications/2007/978924159 5988_eng.pdf (2007).

10. MJ Wawer, F Makumbi and G Kigozi et al, Circumcision in HIV-infected men and its effect on HIV transmission to female partners in Rakai, Uganda: a randomised controlled trial, Lancet 374 (2009), pp. 229-237.

11. JM Baeten, C Celum and TJ Coates, Male circumcision and HIV risks and benefits for women, Lancet 374 (2009), pp. 182-184.

12. Newell M-L and Barnighausen $T$, Male circumcision to cut HIV risk in the general population, Lancet 369 